\title{
Propuesta didáctica para niños con déficit atencional: estimulando el razonamiento analógico verbal para desarrollar la oralidad tardía*
}

\author{
Didactic proposal for children with attention deficit: stimulation of verbal analogical \\ reasoning for developing late orality \\ Proposta para o ensino de crianças com TDAH: \\ incentivando o raciocínio analógico verbal para desenvolver a oralidade tardia
}

\author{
Georgina García Escala ${ }^{1}$, Carlos Ramos Morales ${ }^{2}$ \\ ${ }^{1}$ Universidad de La Serena, 51-204560,gegarcia@userena.cl \\ ${ }^{2}$ Universidad de La Serena, 51-204372, cramos@userena.cl
}

\begin{abstract}
RESUMEN
El déficit atencional es un cuadro neuropsiquiátrico complejo que, cognitivamente, se caracteriza por unos niveles evolutivamente inapropiados en la atención, sobreactividad e impulsividad (Barkley, 2003) y, lingüísticamente, por presentar alteraciones a nivel fonológico, semántico, sintáctico y en sus capacidades discursivas y pragmáticas, en especial en su capacidad para la comprensión oral de significados figurados (Miranda-Casa, YgualFernández y Rosel-Remírez, 2004; Baixauli-Fortea, Roselló y Miranda-Casas, 2004; García y Manghi, 2006). Este desarrollo lingüístico descendido incide no sólo en las habilidades comunicacionales sino que, además, en la comprensión lectora, producción escrita y en el aprendizaje de contenidos curriculares. Por esto, el presente trabajo da a conocer una propuesta didáctica para desarrollar específicamente la capacidad de inferir significados figurados en la lengua oral. La propuesta fue aplicada a 25 escolares de entre 8 a 9 años de edad con déficit atencional, los resultados en este grupo demuestran la efectividad de la propuesta.
\end{abstract}

Palabras clave: déficit atencional, desarrollo tardío de la lengua, comprensión de significados figurados, razonamiento analógico verbal.

\begin{abstract}
Attention deficit disorder is a complex neuropsychiatric manifestation which is cognitively characterized by evolutionarily inappropriate levels of attention, hyperactivity and impulsiveness (Barkley, 2003), and linguistically characterized by the presence of alterations on the phonological, semantic, syntactic, discursive and pragmatic capacities, specially, in the oral comprehension of figurative meaning (Miranda-Casa, Ygual-Fernández y RoselRemírez, 2004; Baixauli-Fortea, Roselló y Miranda-Casas, 2004; García y Manghi, 2006). This inferior linguistic development is not only present in the communicational abilities but also in reading comprehension, written production and the learning of curricular contents. Therefore, this work presents a didactic proposal to develop the capacity to deduce meaning in oral language. The proposal has been applied to 25 students between 8 and 9 years old with attention deficit disorder. The results in this group demonstrate the effectiveness of the proposal.

Key words: attention deficit, belated development of language, understanding of figurative meanings, verbal analogical reasoning.
\end{abstract}

\section{RESUMO}

TDAH é um transtorno neuropsiquiátrico complexo que, cognitivamente, caracteriza-se por níveis desenvolvimento inadequado de atenção, hiperatividade e impulsividade (Barkley, 2003) e, linguisticamente, por deficiências

* Investigación financiada en parte por Proyecto DIULS- PRO8202 
fonológica, sintática, semântica e capacidades discursivas e pragmáticas, sobretudo as relacionadas à capacidade de compreensão oral de significados figurados (Miranda Casa, Ygual-Fernández e Rosel-Remírez, 2004; BaixauliFortea, Roselló e Miranda-Casas, 2004, Garcia e Manghi, 2006). Este desenvolvimento da linguagem não afeta apenas as habilidades de comunicação, mas a compreensão de leitura, escrita e aprendizagem curricular. Nesse sentido, apresenta-se uma abordagem metodológica para o desenvolvimento da capacidade de inferência de significados figurados da linguagem falada, especificamente. A proposta metodológica foi vivenciada por 25 estudantes de 8 e 9 anos, com TDAH e os resultados demonstram a eficácia da proposta.

Palavras chave: TDAH, atraso no desenvolvimento da linguagem, compreensão do significado figurado, raciocínio analógico verbal.

\section{LA EDUCACIÓN COMO PROCESO FORMAL Y RELACIONAL}

Los niños con déficit atencional presentan características cognitivas y lingüísticas muy específicas que acompañan este complejo cuadro neuropsiquiátrico. Si bien el cuadro se manifiesta en el comportamiento y conducta del sujeto, generando desventajas o desadaptación en el ámbito personal, familiar o escolar (Aboitiz y Schrong, 2005), en su cognición se observa unos niveles evolutivamente inapropiados de problemas atencionales, sobreactividad e impulsividad que no pueden explicarse por ningún déficit neurológico importante ni por otros de tipo cognitivo, sensorial, motor o del habla (Barkley, 2003). En su lenguaje, se observan alteraciones en los niveles de análisis del lenguaje (fonológico, semántico, sintáctico) a nivel discursivo y pragmático (Miranda-Casa, Ygual-Fernández y Rosel-Remírez, 2004; Baixauli-Fortea, Roselló y Miranda-Casas, 2004). Se destaca en estos escolares una capacidad de inferenciar significados figurados de la lengua oral más decendido que su grupo de pares que no presenten esta característica (García y Manghi, 2006). Este aspecto crítico del desarrollo del lenguaje tardío incide no sólo en las habilidades comunicacionales e intercambios comunicativos sino que además en la comprensión lectora, producción escrita y en el aprendizaje de contenidos curriculares. Por esto, estimular su desarrollo en estos escolares permitiría desarrollar habilidades compensatorias para prevenir o aminorar en parte dificultades comunicacionales y de aprendizaje escolar.

Ahora bien, la oralidad tardía es el desarrollo lingüístico que ocurre durante la edad escolar. Si bien los niños, cuando ingresan a la escuela, ya han adquirido los niveles estructurales de la lengua a nivel fonético, semántico y sintáctico y utilizan el lenguaje en variadas funciones, en la escuela el lenguaje se complejiza cuantitativa y cualitativamente (Nippold, 1998), puesto que incrementan el nivel léxico y el lenguaje en general se hace más abstracto, así los sujetos se vuelven más capaces para comprender y producir oraciones complejas, pueden realizar inferencias a partir de enunciados figurados y son capaces de adecuar el lenguaje a los contextos comunicativos específicos (Ravid y Tolchinsky, 2002). Este desarrollo de la oralidad tardía se sustenta en habilidades cognitivas y lingüísticas que estarían en la base de dicho desarrollo. Laval (2002) y Crespo (2008) postulan la existencia de habilidades cognitivas y lingüísticas más básicas y centrales, de las cuales depende no sólo el desarrollo de lo oral sino que también el de la lectura y la escritura. Algunas de las habilidades lingüísticas son el razonamiento analógico verbal, la conciencia metapragmática y la teoría de la mente. Por ello, al estimular el desarrollo de una de estas habilidades es probable que tenga un impacto en el nivel de desarrollo de la oralidad.

Es necesario destacar que en los escolares de entre 8 a 9 años de edad, en este desarrollo de la oralidad tardía se produce una especie de salto cuantitativo y cualitativo 
considerable, especialmente en la capacidad de realizar inferencias; probablemente esto ocurra porque estos escolares han consolidado la adquisición de la lectura inicial (Crespo, Benítez y Ramos, 2005). Sin embargo, García y Manghi (2006) han observado que en los niños del mismo nivel de enseñanza pero que presentan déficit atencional, no se manifiesta este salto en el desarrollo lingüístico (García y Manghi, 2006). Más aún, las investigaciones en el área señalan que estos escolares pueden o no presentar alteraciones en los diferentes niveles de análisis del lenguaje; pero que en todos ellos se observan alteraciones importantes a nivel de la estructuración del discurso (Vaquerizo-Madrid, Estévez-Díaz y Pozo-García, 2005), es decir, a nivel pragmático, cuando deben realizar adecuaciones a los contextos comunicativos específicos (Miranda-Casas, Ygual-Fernández, Rosel-Remirez, 2004) y en la capacidad de realizar inferencias de los enunciados figurativos (García y Manghi, 2006; Crespo, García y Montenegro, 2009).

Por el impacto que tiene la oralidad tardía en el aprendizaje escolar no sólo para la adquisición de la lectura y escritura sino que además para comprender los contenidos curriculares que el profesor enseña, el presente trabajo da cuenta de la elaboración y aplicación de una propuesta didáctica para incrementar una habilidad cognitiva básica y central de este desarrollo: el razonamiento analógico verbal. Esta propuesta se aplicó a 25 escolares con déficit atencional con el propósito de observar su incidencia en el nivel de desarrollo del razonamiento analógico y su impacto en la comprensión de significados figurados. El análisis estadístico de los resultados demuestran que la propuesta es efectiva y que, de acuerdo a la teoría, el nivel de razonamiento analógico incide en el nivel de comprensión de significados figurados (Pérez, 2010).

\section{MARCO CONCEPTUAL}

\subsection{DÉFICIT ATENCIONAL}

El déficit atencional es un cuadro neuropsiquiátrico complejo que se manifiesta en la esfera del comportamiento y de la conducta del sujeto, y genera desventaja o desadaptación en distintos ámbitos: personal, familiar, escolar, etc. (Aboitiz y Schrong, 2005). Se caracteriza por unos niveles evolutivamente inapropiados de problemas atencionales, sobreactividad e impulsividad que no pueden explicarse por ningún déficit neurológico importante ni por otros de tipo cognitivo, sensorial, motor o del habla (Barkley, 2003). Este trastorno, que afecta entre un 5 a un $10 \%$ de la población escolar, tiene como origen una disfunción prefrontal (Aboitiz y Schrong, 2005). Por ello, este cuadro se relaciona con dificultades para seguir conductas gobernadas por reglas y con la mantención de conductas de trabajo por períodos de tiempo más o menos extensos (Barkley, 2003).

Las características cognitivas de los sujetos con déficit atencional se relacionan con dificultades en el ámbito de la atención sostenida y de la inhibición conductual. La atención sostenida se refiere a la capacidad para mantener la atención por un tiempo prolongado en un estímulo determinado y la inhibición conductual se refiere a un proceso por medio del cual el sujeto inhibe una respuesta inapropiada (Barkley, 2003). La inhibición conductual requiere de tres procesos muy interrelacionados para implementar esta conducta: la capacidad para inhibir respuestas prepotentes, la capacidad para detener patrones de respuestas habituales y la capacidad para controlar las interferencias (Barkley, 
2003). Los niños con déficit atencional, según Barkley (2003), tienen dificultades en la interrelación de dichos procesos, por eso no logran inhibir una respuesta prepotente que es ineficaz en un contexto particular, a saber, no son capaces de ejercer control sobre sus acciones ni de proteger el tiempo de procesamiento de interrupciones; con ello obtendrían un lapso que les permitiría evaluar a largo plazo la conveniencia o no de dicha acción. Esta característica cognitiva, además, se vincula con la dificultad para ejercer el control motor, es decir, no logran poner en marcha al conjunto de acciones concretas que se requieren para alcanzar su objetivo.

En relación a las características lingüísticas de los niños con déficit atencional, los investigadores concuerdan en señalar que muchos de estos niños presentan un nivel de desarrollo más descendido comparados con aquellos que no padecen este cuadro (MirandaCasa, Ygual-Fernández y Rosel-Remírez, 2004; Baixauli-Fortea, Roselló y Miranda-Casas, 2004). Si bien sus producciones orales espontáneas son excesivas, cuando se les exigen tareas lingüísticas como narrar o dar explicaciones, en las cuales se requiere capacidad de planificar y organizar la conducta verbal, disminuyen considerablemente su respuesta, presentando, además, un discurso poco cohesivo. Una posible explicación a estos fallos sería su falta de habilidad on-line para suprimir la información no relevante y así establecer conexiones correctas; sus habilidades cognitivas no estarían plenamente focalizados en la información necesaria para construir en la memoria una representación estructural más coherente y elaborada del discurso (Miranda-Casa et al., 2004; Baixauli-Fortea et al., 2004). Si bien el grado de dificultad en el desarrollo lingüístico de los escolares con déficit atencional varía de un sujeto a otro, en todos ellos se observa un déficit en las habilidades pragmáticas del lenguaje, ya sea en la conducta dialógica en la cual no logran introducir, mantener ni cambiar el tópico conversacional en forma adecuada, en un uso más rígido del lenguaje y en la capacidad de interpretar los posibles significados de una palabra en contextos diferentes (García et al., 2006). En definitiva, los niños con TDAH parecen ser menos capaces para adaptar su lenguaje a las características de su interlocutor y a los contextos específicos de comunicación.

Es necesario destacar que los niños con déficit atencional que cursan tercer año básico, en comparación con su grupo de pares que no presentan este cuadro, tienen un desarrollo más lento en su capacidad para comprender significados figurados en la lengua oral y en las habilidades cognitivas y metalingüísticas que la sustentan, es decir, en el nivel de desarrollo del razonamiento analógico verbal (García et al., 2006; Crespo, García y Montenegro, 2009). Por la importancia de la oralidad para la lectura y el aprendizaje escolar, se elaboró una propuesta para estimular el desarrollo de una de estas habilidades críticas del desarrollo de la oralidad: el razonamiento analógico.

\subsection{LA ORALIDAD TARDÍA}

El desarrollo de la oralidad comienza al primer año de vida, así, cuando los niños ingresan a la escuela poseen un lenguaje desplegado tanto en los niveles estructurales de la lengua: fonético, semántico y sintáctico como en los aspectos pragmáticos, pero además son capaces de utilizar el lenguaje en variadas funciones y contextos (Ravid y Tolchinsky, 2002). Posteriormente, durante la etapa escolar, cuando se adquiere la lectura y la escritura, la oralidad se vuelve más sofisticada cuantitativa y cualitativamente, el léxico se incrementa y se hace más abstracto, la sintaxis se vuelve más compleja, se 
incrementa la capacidad para realizar inferencias de enunciados figurativos y la capacidad de adecuarse a los contextos comunicativos específicos (Nippold, 1998). En síntesis, se desarrolla la capacidad para pensar en y con el lenguaje, el metalenguaje (Ravid y Tolchinsky, 2002). Por ello, se puede señalar que el desarrollo de la oralidad sirve de base para el desarrollo de la lectura aunque por sí misma no garantiza el éxito en esta tarea. La comprensión oral, por su modalidad específica, se apoya en el contexto inmediato y es factible de solicitar al interlocutor una aclaración frente a un fallo de la comprensión. La comprensión de la lectura, en cambio, es un procesamiento descontextualizado y más abstracto que requiere de otras habilidades cognitivas, sin embargo, se apoya en la oralidad (Cain y Oakhill, 2007).

Si bien, la comprensión de la lengua oral y de la lengua escrita son tareas complejas que involucran diferentes habilidades y procesos cognitivos, existe consenso entre los investigadores en que ambas tareas comparten procesamientos. Estos se dan a nivel de palabra (habilidades fonológicas y decodificación de palabra, de frase (semántica y sintaxis), de texto (habilidades narrativas, tanto en la comprensión como en la producción) y de habilidades metalingüísticas, pensar en y con el lenguaje. Así, para comprender un texto oral o escrito, el oyente/ lector, a nivel de palabras individuales debe descifrar el significado, a nivel de oración debe comprender la estructura sintáctica y el sentido de la frase, a nivel de discurso debe construir un modelo mental del texto y para ello requiere integrar información procedente de distintas oraciones para establecer la coherencia local e incorporar - de la memoria a largo plazo- los conocimientos e ideas necesarios para realizar inferencias de la información implícita y otorgarle un sentido global al texto (Cain et al., 2007).

La oralidad por sí sola no garantiza el éxito en la comprensión de la lectura, también requiere de habilidades cognitivas de orden superior (Cain et al., 2007), pero ambas tareas se interrelacionan mutuamente. Así se ha observado que la consolidación de la lectura y la escritura tiene un fuerte impacto en lo que Nippold (1998) denomina desarrollo tardío de la lengua oral (Crespo et al., 2005). Sin embargo, es necesario destacar que este desarrollo tardío de la oralidad recibe influencias tanto de las habilidades cognitivas como metalingüísticas así como también de un conocimiento del mundo mayor, que la escuela ha proporcionado. Dentro de estas habilidades cognitivas y lingüísticas más básicas y centrales se encuentra el razonamiento analógico verbal, la teoría de la mente y la conciencia metapragmática (Laval, 2002; Crespo, 2008). Por el tema de este trabajo, sólo se analizará la primera habilidad.

\subsection{EL RAZONAMIENTO ANALÓGICO VERBAL}

El razonamiento analógico es un componente central de la cognición humana (Goswami, 1992). Holyoak y Thagard (1995) lo definen como un proceso cognitivo que permite realizar "saltos mentales" entre diferentes dominios, favoreciendo recurrir a información conocida para solucionar o entender un problema nuevo (Vosniadou y Ortony; 1975). Lo que se transfiere en este proceso o "salto mental" desde una situación conocida — llamada "análogo" - a una situación nueva y desconocida — denominada "tópico"- es aquello que es semejante y de interés (Ortony, 1993). El resultado de esta transferencia es una regla que permite identificar semejanzas o diferencias, entender o aclarar una característica, ya sea entre diferentes conceptos (Glynn, 1991) o entre cosas familiares con otras no familiares (Bierker, 2002). 
Los investigadores señalan que el razonamiento analógico está compuesto por dos procesos fundamentales: el acceso al análogo y la extrapolación del análogo al tópico (Clement \& Gentner, 1991; Gentner, 1983, 1989; Gentner \& Gentner, 1983; Gentner \& Lander, 1985; Gick \& Holyoak, 1980, 1983; González Labra, 1997; Holyoak \& Thagard, 1989; Keane, 1988; Medin \& Ortony, 1989; Vosniadou, 1989). Así para acceder al análogo, primero se debe realizar una representación mental del análogo y del tópico, luego identificar y comprender el análogo; después recuperar el análogo; y, finalmente, confirmar la plausibilidad en la relación del análogo con el tópico para superar la incertidumbre acerca de la semejanza entre el tópico y el análogo. Para extrapolar el análogo al tópico, se debe extrapolar la comparación entre la información relevante del análogo al tópico. Por otra parte, Sternberg (1987) señala que el razonamiento analógico está compuesto por seis procesos secuenciales: $1^{\circ}$ ) codificación, se identifican los atributos y los valores de los términos del problema, $2^{\circ}$ ) inferencia, se descubre la regla que relaciona los dos primeros términos, $3^{\circ}$ ) proyección o extrapolación, se infiere la regla de segundo orden que relaciona el primer y tercer término, $4^{\circ}$ ) aplicación, se descubre la regla entre los dos primeros términos para poder generar el último término, $5^{\circ}$ ) respuesta, se prepara y se emite la respuesta y $6^{\circ}$ ) justificación, se emparejan varias alternativas de respuesta con la regla obtenida. Si bien estas propuestas teóricas que explican cómo se realiza una analogía son coincidentes, la propuesta de Sternberg (1987) enfatiza dos procesos, el que se refiere a la recuperación del análogo y el de la justificación.

Esta habilidad cognitiva básica y central de razonar en forma analógica incide en el desarrollo evolutivo, porque es el componente cognitivo que permite descubrir diferencias y similitudes entre diversas entidades para establecer correspondencia entre estructuras y representaciones mentales diferentes a fin de generar nuevas representaciones que facilitan el procesamiento de la información, la resolución de problemas y la comprensión del mundo en general (Gentner, 1983; Carretero et al., 1995; Hummel y Holyoak, 1997). El razonamiento analógico, además, promueve la adquisición de nuevos aprendizajes, el desarrollo de las competencias tardías del lenguaje (Gombert, 1992; Nippold, Erskine \& Freedk, 1998; Levorato y Cacciari, 1992, 1995) y el despliegue del pensamiento creativo (Goswami, 2002). De ahí su importancia en la cognición.

El desarrollo del razonamiento analógico, según algunos investigadores, aparece durante la adolescencia junto a la capacidad de abstracción, capacidad que caracteriza el pensamiento formal (Piaget e Inhelder, 1975). En cambio, para otros autores, el razonamiento analógico aparece más temprano en el desarrollo evolutivo. Ellos observaron que los niños de 3 a 5 años de edad son capaces de transferir conocimientos para resolver problemas, a partir de ejemplos dados en situaciones de aprendizaje (Brown y Kane, 1988; Goswami, 1992 y Bierker, 2002). En esta misma línea, Nippold (1998) señala que, si bien el razonamiento analógico emerge a edades muy tempranas, su refinamiento ocurre en la etapa escolar y en la adolescencia, porque el individuo adquiere mayor conocimiento de mundo y se desarrolla su sistema conceptual, favoreciendo con ello la utilización de los aprendizajes adquiridos y la aplicación de éstos a situaciones nuevas y diversas (Goswami, 1992; 2002). Otro factor que permitiría el refinamiento de este tipo de razonamiento es el desarrollo de estrategias de memoria a largo plazo, posibilitando con ello un aumento en la habilidad para representar múltiples dimensiones (Halford, 1993), cuestión indispensable a la hora de relacionar dos situaciones. 
Finalmente, en el ámbito del lenguaje verbal, el razonamiento analógico permite descubrir relaciones semánticas entre conceptos y transpolarlas a nuevas relaciones conceptuales, favoreciendo con esto el desarrollo de la lectura y la escritura (Gombert, 1992, Nipold, 1998, Levorato y Cacciari, 1995). Además, en el ámbito escolar, el razonamiento analógico está a la base de la adquisición de los conceptos científicos (Goswami, 1992, González, 1997), y humanísticos (Sierra, 1995) que allí se enseñan, ya que le permite a los sujetos describir, exponer, comprender y aprender algo nuevo de dominios diferentes..

Si bien el razonamiento analógico verbal está en la base del desarrollo de la oralidad, de la lectura y del aprendizaje de contenidos curriculares, la enseñanza directa de esta habilidad no es considerada por las escuelas. Así, es probable que los escolares que presentan un menor nivel de desarrollo de la oralidad que su grupo de pares, como los niños con déficit atencional (García y Manghi, 2006), podrían beneficiarse si se les enseñara explícitamente esta habilidad cognitiva que sustenta, entre otras, dicho desarrollo.

\section{PROPUESTA DIDÁCTICA}

La propuesta didáctica para estimular el nivel de desarrollo del razonamiento analógico verbal en niños con déficit atencional de tercer año básico considera, por una parte, los procesos que se requieren para resolver una analogía, por otra, el concepto de enseñanza directa y, por último, el nivel de desarrollo semántico alcanzado por los niños a esta edad.

El razonamiento analógico, como se mencionó anteriormente, es un componente central de la cognición humana (Goswami, 1992) y se lo define como una capacidad para recurrir a información conocida para resolver problemas nuevos (Holyoak et al., 1995; Vosniadou et al., 1975). Si bien lo que se transfiere es aquello que es semejante o de interés (Ortony, 1993), la percepción de estas similitudes en diferentes situaciones y la transferencia de dicha información facilita el aprendizaje y la comprensión (Schiff, Bauminger y Toledo (2009). Se destaca que los investigadores postulan diferentes procesos para llevar a cabo este proceso cognitivo, pero la mayoría concuerda que son dos los procesos centrales: recuperación del análogo y justificación. En el primero, se debe inferir la regla que relaciona los atributos de un problema. En el segundo, se empareja la alternativa de respuesta según la regla obtenida. Este programa para enseñar a razonar en forma analógica considera esta secuencia de pasos básicos.

La propuesta didáctica considera, además, los postulados de la enseñanza directa, es decir, realizar secuencias relevantes y en forma sistemática de los temas a tratar. Para ello, primero se realiza una introducción del nuevo contenido y de las habilidades, luego se realizan prácticas guiadas utilizando la retroalimentación correctiva pero estimulando la autonomía de los niños en la realización de las tareas (Waxman y Walberg, 1999). Si bien, Marzano (2003) señala que no existe una estrategia infalible y ésta debe ser adecuada por el docente a cada contexto en particular, el autor postula seis pasos para la enseñanza de vocabulario. Estos pasos han sido considerados en la secuencia de actividades de este programa: $1^{\circ}$ proporcionar una descripción, explicación, o el ejemplo del nuevo contenido, $2^{\circ}$ invitar a los alumnos a los alumnos a repetir la descripción, explicación o ejemplo en sus propias palabras, $3^{\circ}$ pedir a los alumnos que construyan una imagen, un pictograma $o$ representación simbólica del contenido aprendido, $4^{\circ}$ involucrar a los estudiantes periódicamente en actividades que les ayudan a enriquecer sus conocimientos, $5^{\circ}$ periódicamente 
solicitar a los estudiantes que discutan los contenidos entre sí y $6^{\circ}$ involucrar a los estudiantes periódicamente en los juegos que les permiten jugar con los contenidos aprendidos.

Finalmente, esta propuesta considera el nivel de desarrollo semántico de los niños ya que las primeras relaciones semánticas en aparecer son las de antonimia, posteriormente, las que se relacionan con atributos sensoriales y luego los que se relacionan con la funcionalidad de los objetos. Las últimas categorías semánticas en ser adquiridas se relacionan con aspectos más abstractos como las metonimias e hiperonimias (Owens, 2003). En esta misma línea, Sentis, Nusser y Acuña (2006) señalan que, si bien el desarrollo semántico es considerado por algunos autores como una habilidad prelingüística, este desarrollo se puede observar en que el niño no asigna significados al azar sino que los relaciona primero con atributos sensoriales, luego con causalidad y funcionalidad y más tardíamente con aspectos más abstractos como categoría.

Así, la propuesta para enseñar a razonar en forma analógica promueve la resolución de analogías clásicas o proporcionales en tres ámbitos semánticos: a) antonimia y sinonimia, b) atributivas, causativas, y funcionales y c) metonimia e hiperonimia; ámbitos que según las investigaciones en el área se desarrollan en edades tempranas. Es necesario considerar el nivel de desarrollo semántico de los niños en esta edad ya que para completar una analogía proporcional, el sujeto primero debe establecer la relación semántica entre el primer par de conceptos para luego transpolar dicha relación semántica al segundo par de conceptos. Por ello, en esta propuesta primero se instruye a los niños a descubrir el tipo de relación semántica entre objetos a nivel concreto y, luego, a nivel gráfico para, finalmente, transpolar esta relación semántica a nuevos conceptos, a nivel abstracto. Por ello en esta propuesta se considera, además, la secuencia de concreto a abstracto, según lo postulado por la teoría cognitiva de cómo los sujetos construyen sus conceptos. Por ello, los ejercicios se establecen en el siguiente orden: concreto, gráfico y a nivel de palabra. En cada nivel se exige que los niños verbalicen la tarea cognitiva realizada a fin de asegurarse la comprensión e internalización de ésta. Por esto, dicha propuesta considera cinco etapas para cada tipo de relación semántica a enseñar: $1^{\circ}$ ) enseñar explícitamente la variable a comparar (antónimos, sinónimos, atributivas causativas, funcionales, metonimia, hiponimia o partonimia), $2^{\circ}$ ) aplicar la comparación semántica utilizando elementos concretos, $3^{\circ}$ ) identificar el elemento que no corresponda en una relación semántica dada, para favorecer la flexibilidad cognitiva, $4^{\circ}$ ) aplicar una relación semántica utilizando palabras y $5^{\circ}$ ) completar analogías verbales.

Así, en la primera y segunda etapa se estimula la capacidad cognitiva de los niños para comparar, es decir, para establecer relaciones de cotejo entre objetos concretos, utilizando un criterio semántico. En la tercera etapa se estimula la capacidad para descubrir el elemento que no corresponde en una serie gráfica dada, es decir, debe descubrir la relación semántica entre los objetos y eliminar el que no satisfaga el criterio de comparación. En la cuarta se estimula la capacidad de aplicar la relación semántica trabajada, creando un ejercicio nuevo. En la quinta se enseña a descubrir las relaciones semánticas entre los conceptos y a transpolar dicha relación a nuevos conceptos, es decir, se enseña a razonar en forma analógica. En esta etapa, los ejercicios se realizan con palabras dadas en forma oral y escrita. Finalmente, se realizan una serie de ejercicios para evaluar las dimensiones semánticas trabajadas. Cabe destacar que sólo el ejercicio de la primera etapa (enseñanza de un tipo de relación semántica), y el ejemplo de cada etapa se realiza en forma grupal. El resto de los ejercicios son de realización individual. 
Por último, es necesario señalar que todas las actividades a realizar en esta propuesta son de inducción, es decir, no se debe dar la respuesta correcta, sino que se orienta al niño a que la resuelva por sí mismo, o con la ayuda de sus compañeros. Para una mejor internalización del proceso cognitivo realizado, se solicita a los escolares que luego de responder acertadamente, verbalicen los pasos realizados para resolver la tarea realizada y la escriban o la grafiquen según corresponda. Si bien cada actividad es individual, el grupo puede ayudar en caso de que uno de los sujetos no pueda resolver la tarea dada por el profesor.

\section{METODOLOGÍA}

En esta investigación se trabajó con una muestra inicial de 30 niños de tercer año básico, repartidos en 5 colegios particulares subvencionados de la ciudad de La Serena que presentaban certificación médica de déficit atencional y ausencia de otros trastornos del desarrollo. Es necesario destacar que, en Chile, los colegios que presentan la modalidad de financiamiento compartido -familia y estado- son colegios de nivel socio cultural medio. Esta selección de la muestra obedece a dos razones. Por una parte, se requería de niños que sólo presentaran déficit atencional y ninguna otra alteración que pudiera alterar el nivel de desarrollo evolutivo; por otra, de niños con un nivel sociocultural que garantizara el conocimiento y manejo de un código de habla a nivel de lengua estándar. Posteriormente, al revisar la vigencia de las certificaciones de los estudiantes al finalizar la intervención, la muestra quedó reducida a 25 sujetos.

La propuesta se aplicó por un período de dos meses, durante tres veces a la semana en sesiones de una hora cronológica cada una. Se aplicó en cada colegio durante la jornada escolar del niño, en horarios previamente concertados con el establecimiento educacional para no alterar el proceso de enseñanza de los contenidos curriculares. La modalidad de trabajo fue grupal, de cinco niños con un moderador de la actividad. El trabajo de moderador fue realizado por ayudantes técnicos de investigación.

En esta investigación se aplicaron dos instrumentos como pretest y postest. El Instrumento de Medición de Inferencias Pragmáticas (IMIP) (Crespo et al., 2005) y la Prueba de Razonamiento Analógico Verbal (RAV) (García, 2007). El primero evalúa la capacidad de realizar inferencias a partir de enunciados figurados dados en forma verbal, enunciados como actos de habla indirectos, frases metafóricas e ironías. El segundo, evalúa la capacidad para resolver analogías proporcionales o clásicas con diferentes relaciones semántica en la base: sinónimos, antónimos, atributivas, causativas, funcionales, hiperónimos y metonimia.

\section{ANÁLISIS DE DATOS}

El presente trabajo sintetiza los resultados obtenidos sobre un grupo de escolares con déficit atencional de La Serena que fueron sometidos a un programa de estimulación del razonamiento analógico en el año 2009, con el propósito de mejorar sus niveles de razonamiento analógico verbal (RAV) y de comprensión de significados figurativos (IMIP). Los resultados son presentados por separado para cada una de las pruebas, estimándose los niveles de logro (proporción de respuestas correctas) y los puntajes medios antes y después del programa de estimulación. 


\subsection{PRUEBA RAV}

En la Tabla 1 se presenta el puntaje total en la prueba RAV, por niño, antes y después del programa de estimulación, junto con la diferencia entre ambos puntajes, en tanto que en la Tabla 2 se presentan estadísticos de tendencia central y dispersión de los datos. Por su parte, en la Figura 1 se presentan los puntajes medios en la prueba RAV, junto con los intervalos de confianza antes y después del programa de estimulación. Para determinar si las diferencias en los puntajes son estadísticamente significativas, se aplicó la prueba t para datos pareados (Canavos, 1988). Dicha prueba indica que el valor medio post programa de estimulación en la prueba RAV es significativamente mayor que el puntaje obtenido antes de dicho programa $(\mathrm{t}=4.8616, \mathrm{df}=22$, $\mathrm{p}$-value $=7.37 \mathrm{e}-05)$.

\begin{tabular}{|c|c|c|c|}
\hline ID Individuo & Pre Test & Post Test & Dif \\
\hline $\mathbf{1}$ & 35 & 50 & 15 \\
\hline $\mathbf{2}$ & 35 & 50 & 15 \\
\hline 3 & 35 & 48 & 13 \\
\hline 4 & 38 & 54 & 16 \\
\hline $\mathbf{5}$ & 16 & 52 & 36 \\
\hline $\mathbf{6}$ & 46 & 53 & 7 \\
\hline $\mathbf{7}$ & 45 & 53 & 8 \\
\hline $\mathbf{8}$ & 40 & 50 & 10 \\
\hline $\mathbf{9}$ & 45 & 51 & 6 \\
\hline 10 & 50 & 51 & 1 \\
\hline 11 & 43 & 47 & 4 \\
\hline 12 & 49 & 54 & 5 \\
\hline 13 & 49 & 48 & -1 \\
\hline 14 & 50 & 50 & 0 \\
\hline 15 & 48 & 57 & 9 \\
\hline 16 & 43 & 44 & 1 \\
\hline 17 & 52 & 50 & -2 \\
\hline 18 & 41 & 46 & 5 \\
\hline 19 & 46 & 46 & 0 \\
\hline 20 & 44 & 54 & 10 \\
\hline 21 & 20 & 37 & 17 \\
\hline 22 & 41 & 49 & 8 \\
\hline 23 & 36 & 46 & 10 \\
\hline Promedio & 41 & 50 & 8 \\
\hline Mínimo & 16 & 37 & -2 \\
\hline Máximo & $\mathbf{5 2}$ & $\mathbf{5 7}$ & $\mathbf{3 6}$ \\
\hline & & & \\
\hline
\end{tabular}

Tabla 1. Puntaje RAV según individuo y Diferencia (Dif) entre puntajes Pre y Post

\begin{tabular}{|l|c|c|c|c|c|}
\hline Aplicación & Promedio & Mín & Máx & DesvEst & CV \\
\hline Pre_Test & 41 & 16 & 52 & 9,0 & $22 \%$ \\
\hline Post_Test & 50 & 37 & 57 & 4,2 & $8 \%$ \\
\hline
\end{tabular}

Tabla 2. Resumen de Estadísticas Descriptivas Pre y Post, test RAV 


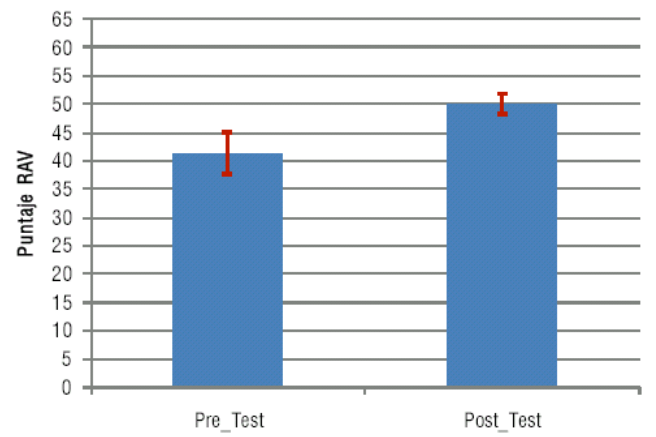

Figura 1. Puntaje medio estimado Pre y Post prueba RAV. Intervalos de confianza del 95\%.

La Figuras 2 y 3 muestran los puntajes antes y después del programa de estimulación por niño; la primera con un gráfico de líneas y la segunda con un diagrama de puntos. En esta se aprecia que 2 niños mostraron un incremento notable en el desempeño en la prueba RAV post el programa de estimulación.

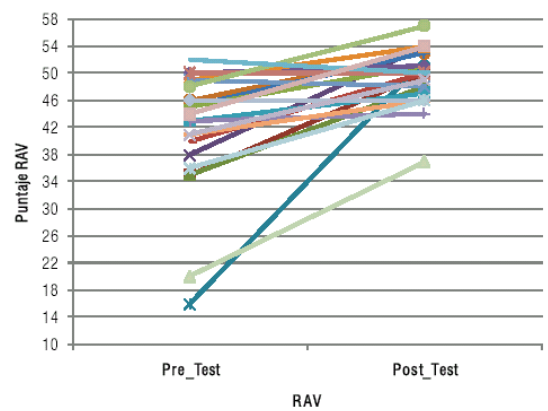

Figura 2. Puntaje Pre y Post, prueba RAV por cada niño (diagrama de líneas).

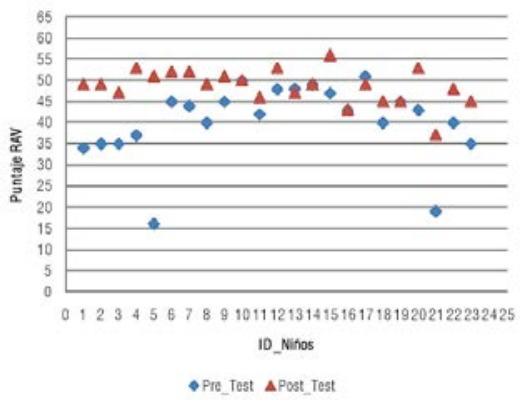

Figura 3. Puntaje Pre y Post, prueba RAV por cada niño (diagrama de puntos). 
Por su parte, la Figura 4 muestra los resultados anteriores a través de un diagrama de dispersión en conjunto con el coeficiente de correlación lineal de Pearson. Todas estas gráficas permiten observar el aumento del puntaje en la prueba RAV a posteriori del programa de estimulación.

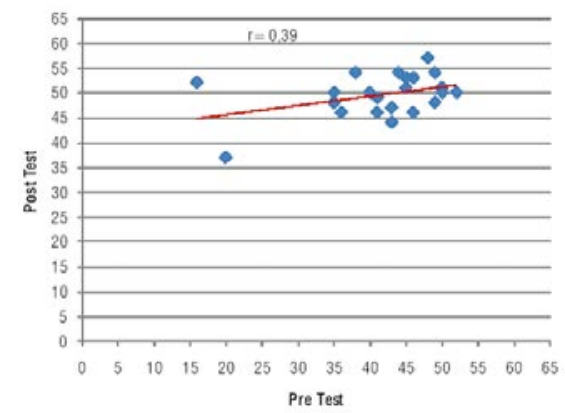

Figura 4. Diagrama de Dispersión entre Puntajes Pre y Post, prueba RAV.

\subsection{PRUEBA IMIP}

En la Tabla 3 se presenta el puntaje total en la prueba IMIP por niño, antes y después del programa de estimulación, junto con la diferencia entre ambos puntajes, en tanto que en la Tabla 4 se presentan estadísticos de tendencia central y dispersión de los datos. Por su parte, en la Figura 5 se presentan los puntajes medios en la prueba IMIP, junto con los intervalos de confianza antes y después del programa de estimulación.

Para determinar si las diferencias en los puntajes son estadísticamente significativas, se aplicó la prueba t para datos pareados (Canavos, 1988). Dicha prueba corrobora que el valor medio post programa de estimulación es significativamente mayor que el puntaje obtenido antes de dicho programa $(\mathrm{t}=5.6758, \mathrm{df}=21$, $\mathrm{p}$-value $=1.239 \mathrm{e}-05)$. 


\begin{tabular}{|c|c|c|c|}
\hline ID_Individuo & Pre_Test & Post_Test & Dif \\
\hline 1 & 32 & 44 & 12 \\
\hline 2 & 32 & 44 & 12 \\
\hline 3 & 33 & 35 & 2 \\
\hline 4 & 35 & 41 & 6 \\
\hline 5 & 27 & 41 & 14 \\
\hline 6 & 38 & 45 & 7 \\
\hline 7 & 35 & 40 & 5 \\
\hline 8 & 37 & 43 & 6 \\
\hline 9 & 43 & 44 & 1 \\
\hline 10 & 36 & 38 & 2 \\
\hline 11 & 30 & 27 & -3 \\
\hline 12 & 43 & 48 & 5 \\
\hline 13 & 35 & 46 & 11 \\
\hline 14 & 39 & 47 & 8 \\
\hline 15 & 42 & 47 & 5 \\
\hline 16 & 42 & 42 & 0 \\
\hline 17 & 45 & 47 & 2 \\
\hline 18 & 39 & 43 & 4 \\
\hline 19 & 39 & 43 & 4 \\
\hline 20 & 42 & 47 & 5 \\
\hline 21 & 42 & 42 & 0 \\
\hline 22 & 38 & 45 & 7 \\
\hline Promedio & 37 & 43 & 5 \\
\hline Mínimo & 27 & 27 & -3 \\
\hline Máximo & 45 & 48 & 14 \\
\hline
\end{tabular}

Tabla 3. Puntaje IMIP según individuo y Diferencia (Dif) entre puntajes Pre y Post

\begin{tabular}{|l|c|c|c|c|c|}
\hline Aplicación & Promedio & Mín & Máx & DesvEst & CV \\
\hline Pre_Test & 37 & 27 & 45 & 4,7 & $13 \%$ \\
\hline Post_Test & 43 & 27 & 48 & 4,7 & $11 \%$ \\
\hline
\end{tabular}

Tabla 4. Resumen de Estadísticas Descriptivas Pre y Post, test IMIP

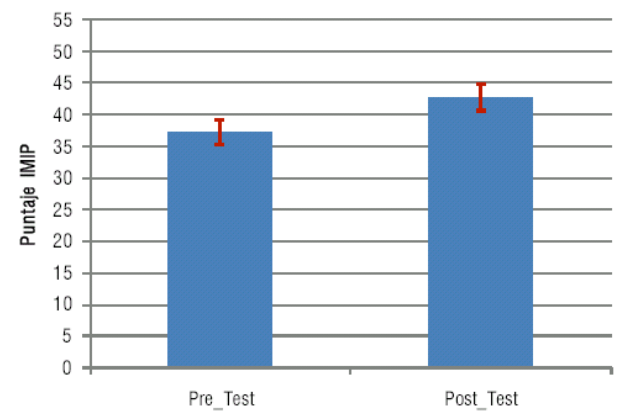

Figura 5. Puntaje medio estimado Pre y Post prueba IMIP. Intervalos de confianza del 95\%. 
Las Figuras 6 y 7 muestran los puntajes antes y después del programa de estimulación por niño; la primera con un gráfico de líneas y la segunda con un diagrama de puntos.

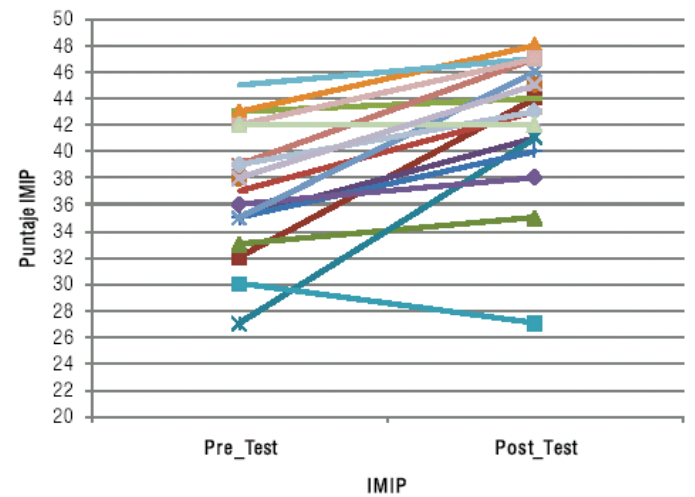

Figura 6. Puntaje Pre y Post, prueba IMIP por cada niño (diagrama de líneas).

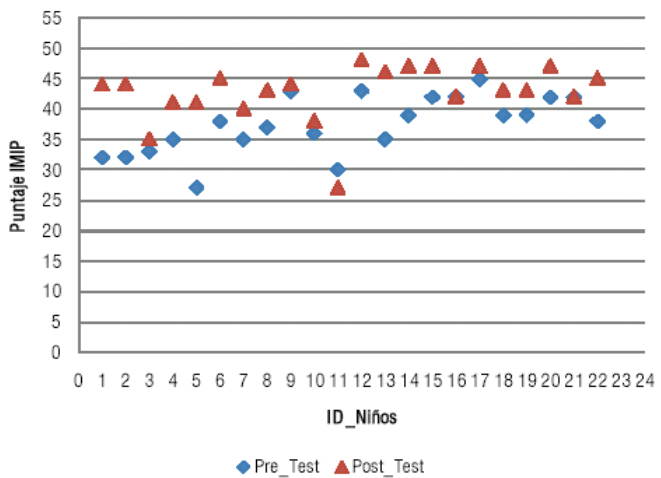

Figura 7. Puntaje Pre y Post, prueba IMIP por cada niño (diagrama de puntos).

Por su parte, la Figura 8 muestra los mismos resultados a través de un diagrama de dispersión en conjunto con el coeficiente de correlación lineal de Pearson. Todas estas gráficas permiten observar el aumento del puntaje en la prueba IMIP a posteriori del programa de estimulación. 


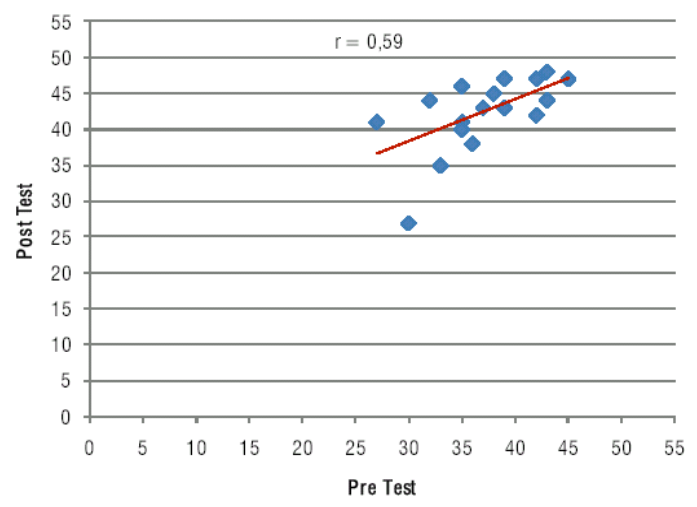

Figura 8. Diagrama de Dispersión entre Puntajes Pre y Post, prueba IMIP.

\section{CONCLUSIONES Y SUGERENCIAS}

Luego del análisis de los datos es posible señalar dos cosas. La primera se refiere a la efectividad de la propuesta didáctica para estimular el razonamiento analógico verbal en niños con déficit atencional de tercer año básico, elaborada en este trabajo. Dicha conclusión se sustenta en que los niños, luego de ser sometidos a esta propuesta didáctica, aumentaron significativamente su nivel de razonamiento analógico verbal. La segunda, se refiere a la comprobación de aspectos teóricos que dan cuenta de las habilidades cognitivas que sustentan el desarrollo de la oralidad. Los niños que fueron sometidos a esta propuesta didáctica, además, aumentaron su nivel de comprensión de significados figurados en la lengua oral. Con ello, no sólo se comprueba que esta propuesta estimula el nivel de desarrollo del razonamiento analógico verbal de los escolares sino que, además, se reafirma lo que postula la teoría, a saber, que esta habilidad cognitiva subyace el desarrollo de un aspecto específico de la oralidad tardía, aquélla que se vincula con la comprensión de significados figurados.

Finalmente, es necesario destacar que la oralidad de los escolares, por su impacto en la lectura y la escritura y en el aprendizaje de contenidos curriculares, debiera ser enseñada por las escuelas en forma explícita. Especialmente, enseñarla a aquellos escolares que presentan alteraciones en el desarrollo evolutivo o que presentan necesidades educativas especiales. De esta manera se favorecería la capacidad de los sujetos de pensar en y con el lenguaje.

Se destaca, además, que este programa de estimulación del razonamiento analógico verbal puede ser utilizado con otras poblaciones escolares especiales que presentan descendida la capacidad de realizar inferencias en el lenguaje, escolares con dislexia, disgrafía, etc.

\section{REFERENCIAS BIBLIOGRÁFICAS}

Austin, J. (1962). How to do things with words. Oxford: University Press.

Aboitiz, F. y Schrong, C. (2005). Síndrome de déficit atencional: antecedentes neurobiológicos y cognitivos para estudiar un modelo de endofenotipo. Revista chilena de Neuro-psiquiatría, vol. 43, n.1, 11-16. 
Baixauli, I., Roselló B. y Miranda, A. (2004). Evaluación de las dificultades pragmáticas. Estudio de casos. Revista Neurológica, vol. 38, n.1, 69-79.

Barkley, R. (1990). Attention déficit hyperactity disorders: A handbook for diagnosis and treatment. New York: Guilford.

Barkley, R. (2003). Issues in the diagnosis of attention-deficit/hyperactivity disorder in children. Brain, vol, 25, 77-83.

Belinchón, M. (1990). Lenguaje no literal y aspectos pragmáticos de la comprensión. En M. de Vega y F. Cuetos (Eds.), Psicolingüística del español (pp. 307-374). Madrid: Trotta.

Bierker, E. (2002). Analogical Thinking: Bringing the past, present, and futureintorelationships. Consultado en junio, 2006, en: http://www.collegetransitiongroup.com/GallagherAnalogyPaper. pdf

Brown, A. y Kane, L. (1998). Preschool children can learn to transfer: Learning to learn and learning from example. Cognitive Psychology, n. 20, 493-523.

Canavos, G.C. (1988). Probabilidad y Estadística, aplicaciones y métodos. México: McGraw Hill/ Interamericana de México.

Carson, R. (2002). Relevance Theory and saying/implicating distinction. In Horn, L. y Ward, G., eds. Handbook of pragmatics psycholinguistics (pp. 123-144). Oxford: Blackwell.

Cain, K. y Oakhill, J. (2007) Children's Comprehension Problems Oral and Written Language a Cognitive Perspective. En Cain, K. y Oakhill, J., eds. Oral and Written Language a Cognitive Perspective. London: Guilford Press.

Carrell P. (1981). Children's understanding of indirect requests: comparingf child and adult comprensión. Journal of Child Language vol. 8, 329-345.

Crespo, N., Benítez, R. y Ramos, C. (2005). Informe Proyecto FONDECYT No 1040740. Valparaíso: Universidad Católica de Valparaíso.

Crespo, N. (2009) La medición de la conciencia metapragmática de los niños: Resolviendo la ambigüedad en la comprensión oral. Revista de Lingüística Teórica y Aplicada, vol. 42, n. 2, 69-88.

Crespo, N., Benítez, R. y Ramos, C. (2005). Una propuesta de medición de las inferencias en la comprensión del discurso oral. En M. Pilleux (Ed.) Los contextos del discurso (pp. 142-151). Valdivia: Asociación Latinoamericana de Estudios del Discurso (ALED), Filial Chile.

Crespo, N., García, G. y Montenegro, C. (2009). La incidencia del déficit atencional en el desarrollo de algunas habilidades cognitivas y metalingüísticas. Revista Sintagma, vol 21, 5-20.

Crespo, N. y Manghi D. (2005) La comprensión del lenguaje oral: elementos para un modelo. Revista Signos, vol 38, n. 59, 269-285.

Escandell, M. (1998). Cortesía y relevancia. En H. Haverkate, G. Mulder y M. Fraile La pragmática lingüística del español: Recientes desarrollos (pp. 237-250). Ámsterdam: Rodopi.

Escandell, M. (1999). Introducción a la pragmática. Barcelona: Ariel.

Etchepareborda, M., Mulas, F., Capillas- González, A., Fernçandez-González, S., Campo, P. y Maestú, F. (2004). Sustrato neurofuncional de la rigidez cognitiva en el trastorno por déficit de atención con hiperactividad: resultados preliminares. Revista Neurología, vol 38, n. 1, 145-48.

García, G. y Manghi, D. (2006) La incidencia del déficit atencional en el desarrollo tardío del lenguaje". Revista de Estudios y Experiencias en Educación, vol. 5, n. 10, 113-125.

García, G. (2007) Razonamiento Analógico Verbal en Escolares: Una propuesta de Medición. Ponencia presentada en el XVII Congreso de la Sociedad Chilena de Lingüística (SOCHIL). Universidad de Concepción.

Gentner, D. (1983) Structure-mapping: A theoretical framework for analogy. Cognitive Science, n. 7, 155-170.

Gibbs, R. (1994) The poetics of mind. Cambridge: University Press.

Gibbs, R. (2002) A new look at literal meaning in understanding what is said and implicated. Journal of Pragmatics, vol 34, 457-486. 
Giora R. (2001). Literal vs. Figurative language. Different or equal? Journal of Pragmatics, vol. $41,240-61$.

Glynn, S. (1991). Explaining science concepts: A teaching-with-analogies-model. En S.M. Glynn, R. Yeany, y B. Britton, (Eds.) The psychology of learning science (pp. 219-240). New York: Hillsdale Erlbaum.

Grice, H. (1975). Logic and conversation. En P. Cole y J.L. Morgan (comp). Syntax and Semantic 3. Speech acts (pp. 41- 58). New York: Academic Press.

Goswami, U. (1992). Analogical reasoning in children. Nueva Jersey: Lawrence Erlbaum Associates. Gombert, J. (1992). Développement métalinguistique. París: Presses Universitaires de France.

Halford, G. (1999). Children's understanding. New York: Lawrence Erlbaum.

Holyoak, K y Thagard, P. (1995). Analogical mapping by constraint satisfaction. Cognitive Science, vol. 13, 295-355.

Hummel, J. y Holyoak, K. (1997) A symbolic-connectionist theory of relational inference and generalization. Psychological Review, vol, 110, 220-264.

Laval, V. (2003) Idiom comprehension and metapragmatic knowledge in French children Journal of pragmatics Source, vol. 35, n. 5, 723-739.

Leinonen, E., Letts, C. y Rae, B. (2002). Children's pragmatic communication difficulties. London: Whur Plublishers.

Levorato, M. y Cacciari, R. (1992) Children's comprehension and production of idioms: The role of context and familiarity. Journal of Child Language, vol. 19, 415-433.

Marzano, R. J. (2003). What works in schools: Translating research into action. Alexandria, VA: Association for Supervision and Curriculum Development.

Miranda, A., Igual- Fernández, A. y Rosel Remírez, J., (2004). Complejidad gramatical y mecanismos de cohesión en la pragmática comunicativa de los niños con trastornos por déficit de atención con hiperactividad. Revista Neurológica, vol. 38, n. 1, 111-16.

Muñoz, J., y Tirapu, J. (2004). Rehabilitación de las funciones ejecutivas. Revista Neurológica, vol 38, n 2, 656-63.

Nippold, M. (1998). Later Language Development: The school age and adolescent years. Texas: Pro. Ed.

Ninio, A Y Snow, C. (1996). Pragmatic development. Boulder CO: Westview Press.

Owens, R. (2003). Desarrollo del lenguaje. Chicago: Pearson.

Ortony, A. (1993). Metaphor and thought. Cambridge: Cambridge University.

Piaget, J. e Inhelder, B. (1975). La psicología del niño. Madrid: Morata.

Pérez, L. (2010). Informe estadístico. Informe interno Proyecto DIULS PRO8202. Universidad de La Serena.

Ravid, D. y Tolcchinsky, L. (2002) Developing Linguistic Literacy: A Comprehensive Mode. Journal of Child Language, vol. 29, n 2, 417-447.

Sánchez, R. y Narbona, J. (2004). El sistema ejecutivo y las lesiones frontales en el niño. Revista neurológica,vol 39, n 2, 188-191.

Schiff, R., Bauminger, N. y Toledo, I. (2009) Analogical Problem Solving in Children with Verbal and Nonverbal Learning Disabilities. Journal of Learning Disabilities, vol 42 n1,3-13.

Searle, J. (1986). Actos de Habla. Madrid: Cátedra.

Sentis, F, Nusser, C. y Acuña, X. (2009). El desarrollo semántico y el desarrollo de la referencia en la adquisición de la lengua materna. Onomazein, vol 20, n. 2, 147-191

Serverá, M. y Gaván, M. (2001). Problemas de impulsividad e inatención en el niño. Madrid: Secretaría General Técnica.

Serverá, M. (2005). Modelo de autorregulación de Barkley aplicado al trastorno por déficit de atención con hiperactividad: una revisión. Revista Neurología, vol. 40, n. 6, 358-368.

Sierra, B. (1995). Inducción y transferencia de esquemas en el procesamiento humano de la información. Tesis doctoral. Madrid: Universidad Autónoma de Madrid.

Sperber, D. y Wilson, D. (1994). La Relevancia. Madrid: Visor. 
Sperber, D. (2000). Metarepresentations: A Multidisciplinary Perspective. Oxford: Oxford University Press.

Stenberg, R. (1987). Inteligencia humana II. Cognición, personalidad e inteligencia. Barcelona: Paidós.

Vaquerizo, J., Estévez, F., y Pozo, A. (2005). El Lenguaje en el trastorno por déficit de atención con hiperactividad: competencias narrativas. Revista Neurología, vol. 40, supl,. 83-89.

Vosniadou, S. y Ortony, A. (1975). Similarity and analogical reasoning: A synthesis. En S. Vosniadou y A. Ortony (Eds.) Similarity and analogical reasoning (pp. 1-17) Cambridge: Cambridge University Press.

Waxman, H. y Walberg, H. (1999). New directions for teaching practice and research. Berkeley, CA: McCutchan Publishing.

Winner, E. y Gardner, H, (1993). Metaphor and irony. En Ortony A, ed. Metaphor and thought (pp. 424-443). Cambridge: Cambridge University Press.

Wilson, D. y Sperber, D. (2002). Relevance theory. En L. Horn y G. Ward (eds.), Handbook of pragmatics psycholinguisticsm (pp. 207-223). Oxford: Blackwell.

Ygual Fernández, A. (2003). Problemas de Lenguaje con estudiantes con déficit atencional. Valencia: Servei de Publicacions. 